\title{
Reflection and transmission of plane harmonic waves at an interface between liquid and micropolar viscoelastic solid with stretch
}

\author{
BALJEET SINGH \\ Mathematics Department, Jat College, Rohtak 124 001, India \\ Address for correspondence: 597/40, Krishnanagar, Hisar 125 001, India \\ e-mail: baljeet_gill@hotmail.com; jangsher@nde.vsnl.net.in \\ MS received 2 November 1999
}

\begin{abstract}
A solution of the field equations governing small motions of a micropolar viscoelastic solid half-space with stretch is employed to study the reflection and transmission at the interface between a liquid and a micropolar viscoelastic solid with stretch. The amplitude ratios for various reflected and refracted waves are computed and depicted graphically. Effects of axial stretch and viscosity on the amplitude ratios are discussed.
\end{abstract}

Keywords. Micropolar viscoelastic solid; axial stretch; reflection coefficient; longitudinal wave; micropolar viscoelastic waves.

\section{Introduction}

The theory of micropolar elasticity constructed by Eringen \& Suhubi (1964) and Eringen (1968) is intended to be applied to materials for problems where the ordinary classical theory of elasticity fails owing to the microstructure of the material. This is the case with regard to problems of grainy and multimolecular bodies. Most of the natural and man-made materials, including engineering, geological and biological media, possess a microstructure. The linear theory of micropolar viscoelasticity was developed by Eringen (1967). Mc Carthy \& Eringen (1969) discussed wave propagation conditions and growth equations. Kumar et al (1990) discussed a plane problem in a micropolar viscoelastic solid with stretch. Many problems of waves and vibrations have been discussed in micropolar elastic solids by several researchers. Notable among them are Parfitt \& Eringen (1969), Tomar \& Gogna (1992, 1995), Tomar \& Kumar (1995) etc. The present paper is concerned with plane wave propagation in an infinite micropolar viscoelastic solid with stretch, and reflection and transmission of plane harmonic waves at the interface between liquid and micropolar viscoelastic solid with stretch. 


\section{Field equations and their solutions}

Following Eringen $(1967,1971)$, the constitutive and field equations of a micropolar viscoelastic solid with stretch (see figure 1), in the absence of body forces and body couples, can be written as

$$
\begin{aligned}
t_{k l} & =\lambda u_{r, r} \delta_{k l}+\mu\left(u_{k, l}+u_{l, k}\right)+\kappa\left(u_{l, k}-\epsilon_{k l r} \phi_{r}\right), \\
m_{k l} & =\beta_{0} \epsilon_{r k l} \Phi_{, r}+\alpha \phi_{r, r} \delta_{k l}+\beta \phi_{k, l}+\gamma \phi_{l, k}, \\
\beta_{k} & =\alpha_{o} \Phi_{, k}+\left(\beta_{o} / 3\right) \epsilon_{r k l} \phi_{r, l},
\end{aligned}
$$

and

$$
\begin{aligned}
& \left(c_{1}^{2}+c_{3}^{2}\right) \nabla(\nabla \cdot \mathbf{u})-\left(c_{2}^{2}+c_{3}^{2}\right) \nabla \times(\nabla \times \mathbf{u})+c_{3}^{2} \nabla \times \boldsymbol{\phi}=\ddot{\mathbf{u}}, \\
& \left(c_{4}^{2}+c_{5}^{2}\right) \nabla(\nabla \cdot \boldsymbol{\phi})-c_{4}^{2} \nabla \times(\nabla \times \boldsymbol{\phi})+\omega_{o}^{2} \nabla \times \mathbf{u}-2 \omega_{o}^{2} \boldsymbol{\phi}=\ddot{\boldsymbol{\phi}}, \\
& \alpha \nabla^{2} \Phi-\eta_{o} \Phi=(\rho j / 2) \ddot{\Phi},
\end{aligned}
$$

where

$$
\begin{array}{lll}
c_{1}^{2}=(\lambda+2 \mu) / \rho, & c_{2}^{2}=\mu / \rho, & c_{3}^{2}=\kappa / \rho, \\
c_{4}^{2}=\gamma / \rho j, & c_{5}^{2}=(\alpha+\beta) / \rho j, & \omega_{o}^{2}=c_{3}^{2} / j=\kappa / \rho j, \\
\lambda=\lambda^{*}+(\partial / \partial t) \lambda_{v}^{*}, & \mu=\mu^{*}+(\partial / \partial t) \mu_{v}^{*}, & \kappa=\kappa^{*}+(\partial / \partial t) \kappa_{v}^{*}, \\
\alpha=\alpha^{*}+(\partial / \partial t) \alpha_{v}^{*}, & \beta=\beta^{*}+(\partial / \partial t) \beta_{v}^{*}, & \gamma=\gamma^{*}+(\partial / \partial t) \gamma_{v}^{*}, \\
\nabla=\hat{i} \frac{\partial}{\partial x}+\hat{k} \frac{\partial}{\partial z}, &
\end{array}
$$

$\lambda^{*}, \mu^{*}, \kappa^{*}, \alpha^{*}, \beta^{*}, \gamma^{*}, \lambda_{v}^{*}, \mu_{v}^{*}, \kappa_{v}^{*}, \alpha_{v}^{*}, \beta_{v}^{*}, \gamma_{v}^{*}, \alpha_{v}^{*}, \beta_{v}^{*}, \eta_{0}$ are material constants, $\rho$ is density, $j$ is rotational inertia and $\Phi$ is scalar microstretch. $\mathbf{u}$ and $\phi$ are displacement and microrotation vectors respectively. Superposed dots on the right hand side of (4) and (5) stand for second partial derivative with respect to time.

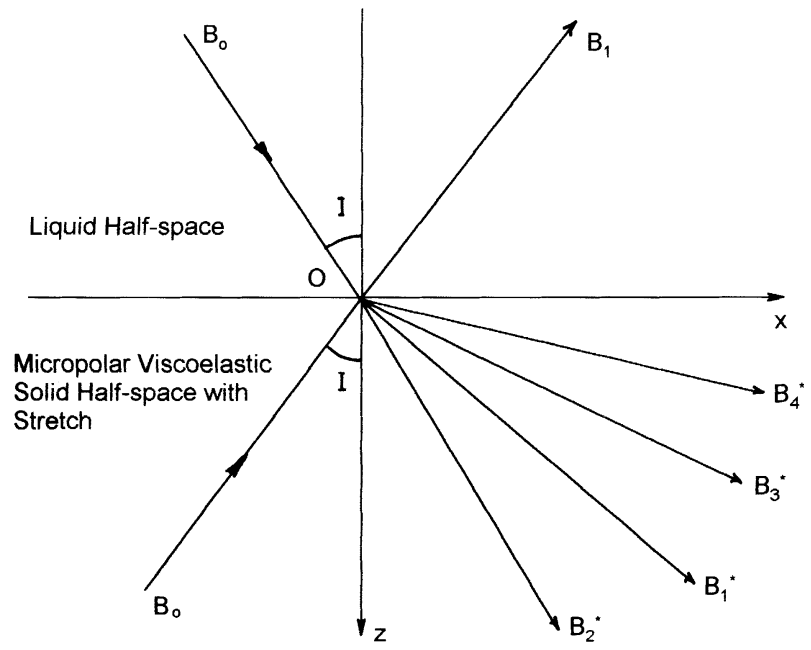

Figure 1. Geometry of the problem. 
Taking $\mathbf{u}=\left(u_{1}, 0, u_{3}\right)$ and $\boldsymbol{\phi}=\left(0, \phi_{2}, 0\right)$ and introducing potentials $\phi(x, z, t)$ and $\psi(x, z, t)$ which are related to displacement components, we get

$$
u_{1}=\frac{\partial \phi}{\partial z}+\frac{\partial \psi}{\partial x}, \quad u_{3}=\frac{\partial \phi}{\partial x}-\frac{\partial \psi}{\partial z}
$$

Substituting the displacement components given by (8) in (4) to (6), we obtain

$$
\begin{aligned}
& \left(\nabla^{2}-\frac{1}{\left(c_{1}^{2}+c_{3}^{2}\right)} \frac{\partial^{2}}{\partial t^{2}}\right) \phi=0 \\
& \left(\nabla^{2}-\frac{1}{\left(c_{2}^{2}+c_{3}^{2}\right)} \frac{\partial^{2}}{\partial t^{2}}\right) \psi-p \phi_{2}=0 \\
& \left(\nabla^{2}-2 q-\frac{1}{c_{4}^{2}} \frac{\partial^{2}}{\partial t^{2}}\right) \phi_{2}+q \nabla^{2} \psi=0, \\
& \left(\nabla^{2}-r_{1}-\frac{1}{c_{6}^{2}} \frac{\partial^{2}}{\partial t^{2}}\right) \Phi=0
\end{aligned}
$$

where

$$
p=\mu /(\mu+\kappa), \quad q=\kappa / \gamma, \quad c_{6}^{2}=2 \alpha_{o} / \rho j, \quad r_{1}=\eta_{0} / \alpha_{0} .
$$

Assuming harmonic time variation as

$$
\begin{aligned}
& \phi(x, z, t)=\bar{\phi}(x, z) \exp (i \omega t), \\
& \psi(x, z, t)=\bar{\psi}(x, z) \exp (i \omega t), \\
& \phi_{2}(x, z, t)=\bar{\phi}_{2}(x, z) \exp (i \omega t), \\
& \Phi(x, z, t)=\bar{\Phi}(x, z) \exp (i \omega t) .
\end{aligned}
$$

Substituting (14) in (9) to (12), we get

$$
\begin{aligned}
& \left(\nabla^{2}+\frac{\omega^{2}}{V_{1}^{2}}\right) \bar{\phi}=0, \\
& \left(\nabla^{4}+\omega^{2} B \nabla^{2}+\omega^{4} C\right)\left(\bar{\psi}, \bar{\phi}_{2}\right)=0, \\
& \left(\nabla^{2}+\frac{\omega^{2}}{V^{2}}\right) \bar{\Phi}=0,
\end{aligned}
$$

where

$$
\begin{aligned}
& B=\frac{q(p-2)}{\omega^{2}}+\frac{1}{\left(c_{2}^{2}+c_{3}^{2}\right)}+\frac{1}{c_{4}^{2}}, \\
& C=\frac{1}{\left(c_{2}^{2}+c_{3}^{2}\right)}\left(\frac{1}{c_{4}^{2}}-\frac{2 q}{\omega^{2}}\right),
\end{aligned}
$$

and

$$
V^{2}=c_{6}^{2} /\left(1-\frac{r_{1} c_{6}^{2}}{\omega^{2}}\right), \quad V_{1}^{2}=c_{1}^{2}+c_{3}^{2} .
$$


In an unbounded medium, the solution of (15) corresponds to a modified longitudinal displacement wave propagating with velocity $V_{1}$. The solution of (16) can be written as

$$
\bar{\psi}=\bar{\psi}_{1}+\bar{\psi}_{2}
$$

where $\psi_{1}$ and $\psi_{2}$ satisfy

$$
\begin{aligned}
& \left(\nabla^{2}+\delta_{1}^{2}\right) \bar{\psi}_{1}=0, \\
& \left(\nabla^{2}+\delta_{2}^{2}\right) \bar{\psi}_{2}=0,
\end{aligned}
$$

and where

$$
\begin{aligned}
& \delta_{1}^{2}=\lambda_{1}^{2} \omega^{2}, \quad \delta_{2}^{2}=\lambda_{2}^{2} \omega^{2}, \\
& \lambda_{1,2}^{2}=\left[B \pm\left(B^{2}-4 C\right)^{1 / 2}\right] / 2 .
\end{aligned}
$$

From (10) we obtain

$$
\bar{\phi}_{2}=E \bar{\psi}_{1}+F \bar{\psi}_{2}
$$

where

$$
E=\left(\frac{\omega^{2}}{c_{2}^{2}+c_{3}^{2}}-\delta_{1}^{2}\right) / p, \quad F=\left(\frac{\omega^{2}}{c_{2}^{2}+c_{3}^{2}}-\delta_{2}^{2}\right) / p .
$$

Thus there are two waves propagating with velocities $\lambda_{1}^{-1}$ and $\lambda_{2}^{-1}$ each consisting of transverse displacement $\psi$ and transverse microrotation $\phi_{2}$. Following Parfitt \& Eringen (1969), we call these waves the modified coupled transverse displacement wave and the transverse microrotational wave, respectively.

Equation (17) shows that there exists a wave propagating with velocity $V$, which we call a longitudinal microstretch wave in a micropolar viscoelastic medium with stretch.

This velocity $V$ is real and finite if

$$
1-\left(r_{1} c_{6}^{2} / \omega^{2}\right)>0 \text {. }
$$

The inequality (26) with the help of (13) reduces to

$$
\omega>\omega_{c},
$$

where

$$
\omega_{c}=2^{1 / 2} \eta_{0} / \rho j .
$$

This is the condition for the existence of a modified longitudinal microstretch wave.

For the liquid half-space, the equation of motion in terms of displacement $\phi^{\prime}$ is given by

$$
\nabla^{2} \phi^{\prime}=\frac{1}{\alpha^{2}} \ddot{\phi}^{\prime},
$$

where $\alpha^{\prime 2}\left(=\lambda^{\prime} / \rho^{\prime}\right)$ is the square of the velocity of sound in liquid.

The displacement components $u_{1}^{\prime}, u_{3}^{\prime}$ and pressure $p^{\prime}$ are given by

$$
u_{1}^{\prime}=\frac{\partial \phi^{\prime}}{\partial x}, \quad u_{3}^{\prime}=\frac{\partial \phi^{\prime}}{\partial z} \quad \text { and } \quad p^{\prime}=-\rho^{\prime} \ddot{\phi}^{\prime} .
$$




\section{Boundary conditions}

For two-dimensional motion, the boundary conditions appropriate at the interface $z=0$, are the continuity of normal force stress and normal displacement and the vanishing of the tangential force stress, tangential couple stress and vector first moment across the interface $z=0$; that is

$$
t_{z z}=-p^{\prime}, t_{z x}=0, m_{z y}=0, u_{3}=u_{3}^{\prime}, \beta_{z}=0, \text { at } z=0 .
$$

\section{Reflection and refraction}

We consider the propagation of plane waves in the $x z$-plane, incident at an angle $I$ with the normal to the boundary. For an incident longitudinal wave, $c=\alpha^{\prime} \operatorname{cosec} I$; for an incident modified longitudinal displacement wave, $c=V_{1} \operatorname{cosec} I$; for an incident set of modified coupled transverse displacement and microrotational waves, $c=\lambda_{1}^{-1} \operatorname{cosec} I$ and for an incident longitudinal microstretch wave, $c=V \operatorname{cosec} I$; where $c$ is the apparent phase velocity on the surface.

When a micropolar wave modified due to viscous and stretch effects (i.e. a modified longitudinal displacement wave or one of the sets of modified coupled transverse displacement and microrotational waves or a longitudinal microstretch wave) is incident at the interface $z=0$, we get four reflected waves. A modified longitudinal displacement wave, two sets of two modified coupled waves and a longitudinal microstretch wave in micropolar viscoelastic medium with stretch $(z>0)$ and a refracted longitudinal wave in the liquid medium $(z<0)$. Corresponding to the incident longitudinal wave propagating through the liquid half-space, we obtain a reflected longitudinal wave in the liquid halfspace and four waves as refracted waves in micropolar viscoelastic solid half-space with stretch. The complete geometry showing these reflected and transmitted waves has been shown in figure 1.

The potential functions $\{$ after leaving $\exp i(\omega t-k x)\}$ are as follows:

$$
\begin{aligned}
\phi^{\prime} & =B_{0} \exp \left(i d_{1}^{\prime} z\right)+B_{1} \exp \left(-i d_{1}^{\prime} z\right), \\
\phi & =B_{0} \exp (-i d \beta z)+B_{1}^{*} \exp (i d \beta z), \\
\psi & =B_{0} \exp \left(-i d \alpha_{1} z\right)+B_{2}^{*} \exp \left(i d \alpha_{1} z\right)+B_{3}^{*} \exp \left(i d \alpha_{2} z\right), \\
\phi_{2} & =E B_{0} \exp \left(-i d \alpha_{1} z\right)+E B_{2}^{*} \exp \left(i d \alpha_{1} z\right)+F B_{3}^{*} \exp \left(i d \alpha_{2} z\right), \\
\Phi & =B_{0} \exp \left(-i d_{1} z\right)+B_{4}^{*} \exp \left(i d_{1} z\right),
\end{aligned}
$$

where

$$
\begin{aligned}
& d_{1}^{\prime}=k\left\{\left(c / \alpha^{\prime}\right)^{2}-1\right\}^{1 / 2}, \quad d \beta=k\left\{\left(c / V_{1}\right)^{2}-1\right\}^{1 / 2}, \quad d \alpha_{1}=k\left\{\left(c \lambda_{1}\right)^{2}-1\right\}^{1 / 2}, \\
& d \alpha_{2}=k\left\{\left(c \lambda_{2}\right)^{2}-1\right\}^{1 / 2}, \quad d_{1}=k\left\{(c / V)^{2}-1\right\}^{1 / 2},
\end{aligned}
$$

and for an incident longitudinal wave, $B_{0}=0$ in (31) to (34); for an incident modified longitudinal displacement wave, $B_{0}$ is zero in (30) and (32) to (34); for an incident set of modified coupled waves, $B_{0}$ is zero in (30), (31) and (34) and for incident modified longitudinal microstretch wave, $B_{0}$ is zero in (30) to (33). 
Making use of the potentials given by (30) to (34) in boundary conditions (29) after using (1) to (3), (8) and (29), we obtain the following system of nonhomogeneous equations

$$
\sum_{i=1}^{5} a_{i j} Z_{j}=b_{i},
$$

where $a_{i j}$ are

$$
\begin{aligned}
& a_{11}=-\rho^{\prime} \omega^{2}, \quad a_{12}=\lambda\left(d \beta^{2}+k^{2}\right)+(2 \mu+\kappa) d \beta^{2}, \\
& a_{13}=(2 \mu+\kappa) k d \alpha_{1}, \quad a_{14}=(2 \mu+\kappa) k d \alpha_{2}, \quad a_{15}=0, \\
& a_{21}=0, \quad a_{22}=(2 \mu+\kappa) k d \beta, \\
& a_{23}=\left\{\mu\left(k^{2}-d \alpha_{1}^{2}\right)-\kappa\left(d \alpha_{1}^{2}+E\right)\right\}, \\
& a_{24}=\left\{\mu\left(k^{2}-d \alpha_{2}^{2}\right)-\kappa\left(d \alpha_{2}^{2}+F\right)\right\}, \quad a_{25}=0, \\
& a_{31}=0=a_{32}, \quad a_{33}=\gamma E d \alpha_{1}, \quad a_{34}=\gamma F d \alpha_{2}, \quad a_{35}=\beta_{0} k, \\
& a_{41}=-d_{1}^{\prime}, \quad a_{42}=-d \beta, \quad a_{43}=a_{44}=-k, \quad a_{45}=0, \\
& a_{51}=0=a_{52}, \quad a_{53}=-k E \beta_{0} / 3, \quad a_{54}=-k F \beta_{0} / 3, \quad a_{55}=\alpha_{0} d_{1},
\end{aligned}
$$

and, $b_{i j}$ are related to $a_{i j}$ as

(a) incident longitudinal wave -

$$
b_{1}=-a_{11}, b_{2}=a_{21}, b_{3}=-a_{31}, b_{4}=a_{41}, b_{5}=a_{51},
$$

(b) incident modified longitudinal displacement wave -

$$
b_{1}=-a_{12}, b_{2}=a_{22}, b_{3}=-a_{32}, b_{4}=a_{42}, b_{5}=a_{52},
$$

(c) incident modified coupled wave -

$$
b_{1}=a_{13}, b_{2}=-a_{23}, b_{3}=a_{33}, b_{4}=-a_{43}, b_{5}=a_{53},
$$

(d) incident longitudinal microstretch wave -

$$
b_{1}=a_{15}, b_{2}=a_{25}, b_{3}=-a_{35}, b_{4}=a_{45}, b_{5}=a_{55},
$$

and $Z_{i}(i=1,2, \ldots, 5)$ are the amplitude ratios for various reflected and refracted waves.

If we neglect both viscous and stretch effects, we obtain the system of equations for the problem of reflection and transmission of plane waves at the interface between liquid and micropolar elastic solid. Further, if we remove the liquid half-space, we obtain the system of equations for the problem of reflection of plane waves from the free surface of micropolar elastic solid which agrees with those obtained by Parfitt \& Eringen (1969).

\section{Numerical results and discussion}

Theory indicates that the amplitude ratios $\left|Z_{i}\right|,(1,2, \ldots, 5)$ depend on the angle of incidence of the incident wave. To study these in greater detail, the dependence of these ratios on properties of media together with the angle of incidence, we compute the amplitude ratios. We take the case of aluminium-epoxy composite subject to viscous effect 
and stretch effect for our calculations. Following Gauthier (1982), the physical constants used for micropolar elastic solids are

$$
\begin{aligned}
& \lambda^{*}=7.59 \times 10^{10} \mathrm{~N} / \mathrm{m}^{2}, \quad \mu^{*}=1.89 \times 10^{10} \mathrm{~N} / \mathrm{m}^{2}, \\
& k^{*}=1.49 \times 10^{8} \mathrm{~N} / \mathrm{m}^{2}, \quad \rho=2.19 \times 10^{3} \mathrm{~kg} / \mathrm{m}^{3}, \\
& \gamma^{*}=2.68 \times 10^{4} \mathrm{~N}, \quad j=1.96 \times 10^{-6} \mathrm{~m}^{2}, \quad \omega^{2} / \omega_{0}^{2}=200 .
\end{aligned}
$$

For a particular model of micropolar viscoelastic solid with stretch, the physical constants are given as

$$
\begin{array}{ll}
\lambda=\lambda^{*}\left(1+i Q_{1}^{-1}\right), & \mu=\mu^{*}\left(1+i Q_{2}^{-1}\right), \\
\kappa=\kappa^{*}\left(1+i Q_{3}^{-1}\right), & \gamma=\gamma^{*}\left(1+i Q_{4}^{-1}\right),
\end{array}
$$

where $Q_{i}(i=1,2, \ldots, 4)$ are chosen arbitrarily as

$$
Q_{1}=5, \quad Q_{2}=10, \quad Q_{3}=15, \quad Q_{4}=13,
$$

and

$$
\alpha_{0}=9.15 \times 10^{5} \mathrm{~N}, \quad \beta_{0}=7.26 \times 10^{5} \mathrm{~N}, \quad \eta_{0}=5.32 \times 10^{5} \mathrm{~N} .
$$

Relevant parameters for the liquid half-space are

$$
\rho^{\prime}=1000 \mathrm{~kg} / \mathrm{m}^{3}, \quad \lambda^{\prime}=2.21 \times 10^{10} \mathrm{~N} / \mathrm{m}^{2} .
$$

For the above values of relevant physical constants, the system of equations (35) are solved for amplitude ratios by the application of the Gauss elimination method for different angles of incidence varying from $0^{\circ}$ to $90^{\circ}$. The variation of the amplitude ratios with the angle of incidence is shown graphically in figure $2-11$. Here, we discuss the numerical results in detail, for the cases of incident longitudinal wave and incident modified longitudinal displacement wave. The solid lines in the figures represent variations without viscous and stretch effects whereas dotted lines $L_{1}$ and $L_{2}$ represent variations due to the viscous effect only and due to both viscous and stretch effects respectively.

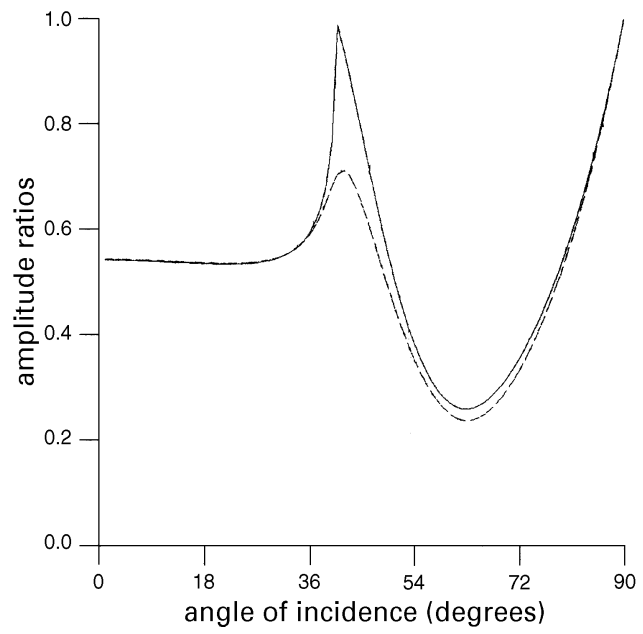

Figure 2. Variations of the modulus of amplitude ratios with the angle of incidence, for reflected longitudinal waves. 


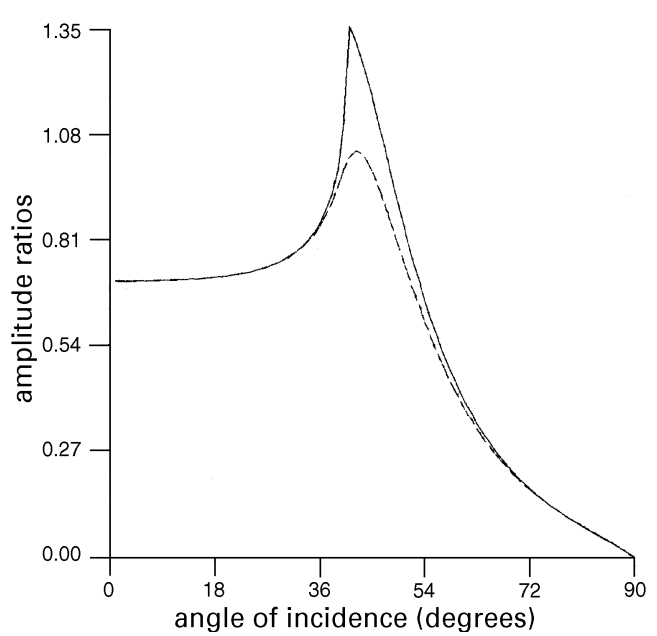

Figure 3. Variations of the modulus of amplitude ratios with the angle of incidence, for refracted modified LD waves.

\section{Case (1) - Incident longitudinal wave}

The variation of amplitude ratios $\left|Z_{i}\right|,(1,2, \ldots, 5)$ with the angle of incidence $I$ of the incident longitudinal wave starting from $0^{\circ}$ (grazing incidence) to $90^{\circ}$ (normal incidence) is depicted in figures 2 to 6 . It is evident that the reflection and refraction coefficients depend on the angle of incidence, the nature of dependence on the angle of incidence is, however, different for different reflected and transmitted waves.

The amplitude ratio $\left|Z_{1}\right|$ for the reflected longitudinal wave remains almost constant (figure 2) for the range $0^{\circ} \leq I \leq 33^{\circ}$ but increases sharply thereafter. It decreases sharply again for the range $41^{\circ} \leq \theta_{o} \leq 63^{\circ}$ and attains a minimum near $I=63^{\circ}$. Beyond $I=63^{\circ}$, it increases sharply again to its maximum near $I=90^{\circ}$. This is shown graphically by the dotted line $L_{2}$ in figure 2 . If we compare the solid line with the dotted lines $\left(L_{1}\right.$ and $\left.L_{2}\right)$, we find that the amplitude ratio decreases slightly due to viscous and stretch effects.

Variation of the amplitude ratio $\left|Z_{2}\right|$ for refracted modified longitudinal displacement wave has been depicted by the dotted line $L_{2}$ in figure 3 . It attains its maximum near

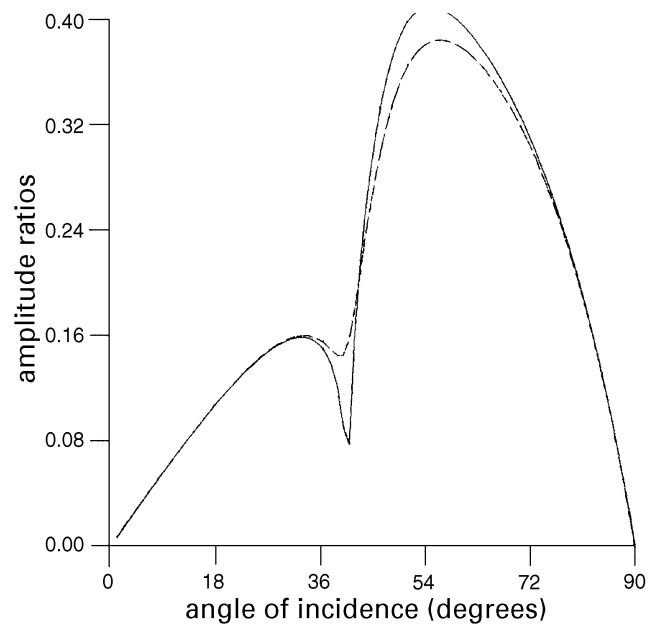

Figure 4. Variations of the modulus of amplitude ratios with the angle of incidence, for refracted modified CDI waves. 


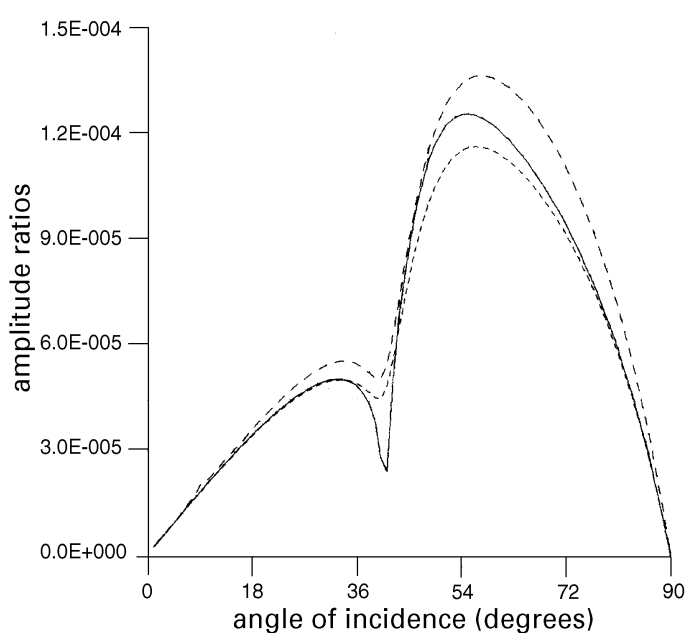

Figure 5. Variations of the modulus of amplitude ratios with the angle of incidence, for refracted modified CDII waves.

$I=41^{\circ}$ and sharply decreases to its minimum at $I=90^{\circ}$. If we compare the solid line with the dotted lines $\left(L_{1}\right.$ and $\left.L_{2}\right)$, we find that the amplitude ratio decreases due to viscous and stretch effects.

Variation of the amplitude ratios $\left|Z_{3}\right|$ and $\left|Z_{4}\right|$ for refracted modified coupled waves have been shown by the dotted lines $\left(L_{2}\right)$ in figures 4 and 5 . Amplitude ratios $\left|Z_{3}\right|$ and $\left|Z_{4}\right|$ for CD I and CD II waves show monotonic variations and attain their respective maxima near $I=55^{\circ}$. On comparing solid and dotted lines $\left(L_{1}\right.$ and $\left.L_{2}\right)$, we observe the effect of viscous and stretch effects on these coupled waves.

Variation of the amplitude ratio $\left|Z_{5}\right|$ for refracted longitudinal microstretch (LMS) wave is shown by the dotted line $L_{2}$ in figure 6 . The variation of the amplitude ratio $\left|Z_{5}\right|$ is monotonic and attains its maximum near $I=55^{\circ}$. If we neglect stretch effect, this wave will not appear.

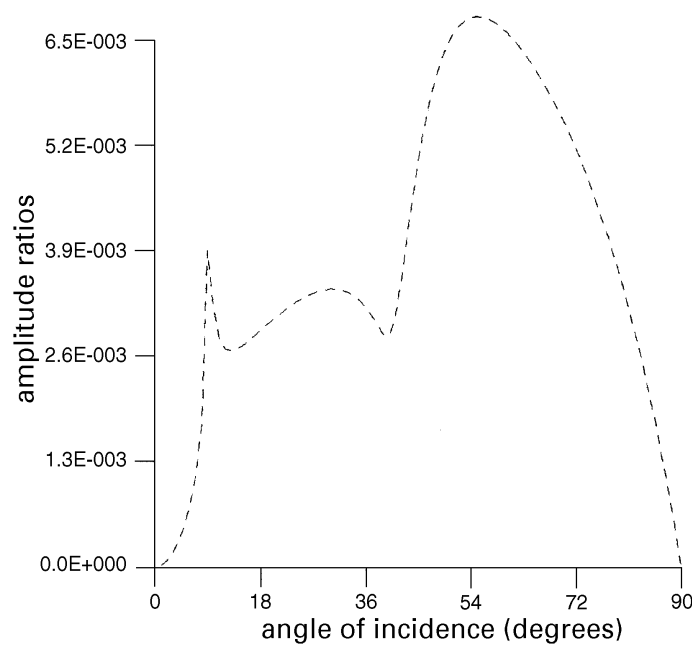

Figure 6. Variations of the modulus of amplitude ratios with the angle of incidence, for refracted LMS waves. 


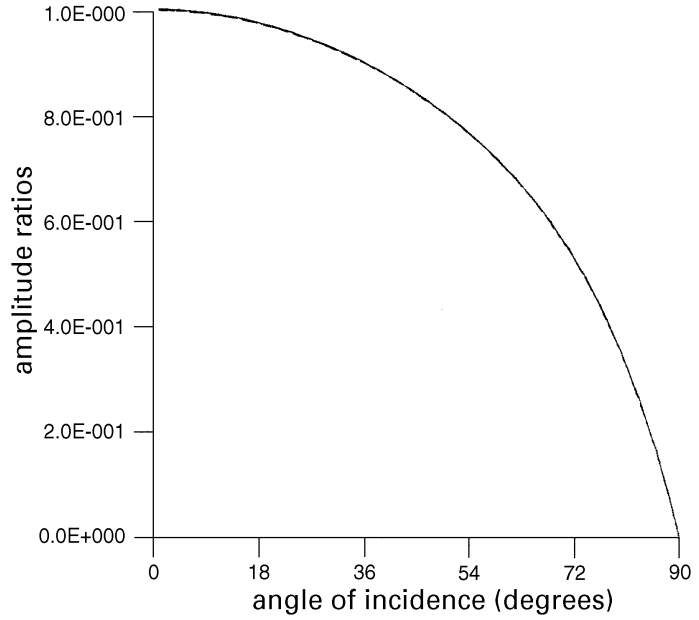

Figure 7. Variations of the modulus of amplitude ratios with the angle of incidence, for refracted longitudinal waves.

\section{Case (2) - Incident modified longitudinal displacement wave}

Variation of amplitude ratios $\left|Z_{i}\right|,(1,2, \ldots, 5)$ with the angle of incidence $I$ of the incident modified longitudinal displacement wave starting from $I=0^{\circ}$ (grazing incidence) to $90^{\circ}$ (normal incidence) are depicted in figures 7 to 11.

The amplitude ratio $\left|Z_{1}\right|$ for refracted longitudinal wave decreases slowly to its minimum near $I=55^{\circ}$ but it increases sharply to its maximum at $I=90^{\circ}$. The variation of this amplitude ratio is shown by the dotted line $L_{2}$ in figure 7 . If we compare the solid line with the dotted lines $\left(L_{1}\right.$ and $\left.L_{2}\right)$, we find that the amplitude ratio changes slightly due to the viscous and stretch effects.

The variation of the amplitude ratio $\left|Z_{2}\right|$ for reflected modified longitudinal displacement wave has been depicted by the dotted line $L_{2}$ in figure 8 . It decreases from its maximum at $I=0^{\circ}$ to its minimum at $I=90^{\circ}$. If we compare the solid line with the dotted lines $\left(L_{1}\right.$ and $L_{2}$ ), we find that the viscous and stretch effects are not significant.

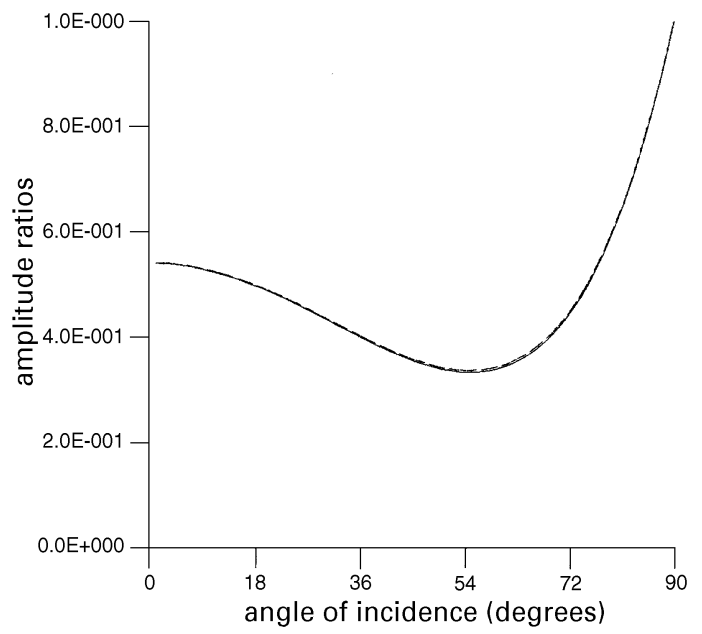

Figure 8. Variations of the modulus of amplitude ratios with the angle of incidence, for reflected LD waves. 


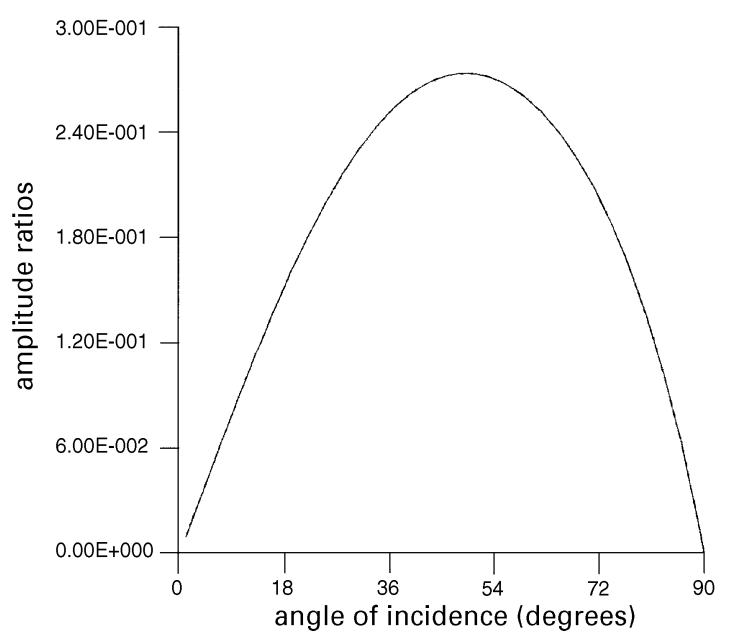

Figure 9. Variations of the modulus of amplitude ratios with the angle of incidence, for reflected modified CDI waves.

Variation of the amplitude ratios $\left|Z_{3}\right|$ and $\left|Z_{4}\right|$ for reflected modified coupled waves is shown by dotted lines $\left(L_{2}\right)$ in figures 9 and 10 respectively. The amplitude ratios $\left|Z_{3}\right|$ and $\left|Z_{4}\right|$ for CD I and CD II waves first increase to their respective maxima near $I=49^{\circ}$ and then decrease to zero at $I=90^{\circ}$. On comparing the solid and the dotted lines $\left(L_{1}\right.$ and $\left.L_{2}\right)$, we observe the effect of viscous and stretch effects on these coupled waves.

Variation of the amplitude ratio $\left|Z_{5}\right|$ for reflected longitudinal microstretch (LMS) waves is shown by the dotted line $L_{2}$ in figure 11 . The amplitude ratio $\left|Z_{5}\right|$ increases sharply to its maximum near $I=15^{\circ}$. Beyond this, it shows a monotonic fall. This wave will not exist in the absence of axial stretch effect.

\section{Conclusions}

The equations of motion for micropolar viscoelastic solid with stretch in the absence of body forces and couples have been analysed. The solutions lead to the existence of a new wave which we call as longitudinal microstretch wave. The existence of this stretch wave

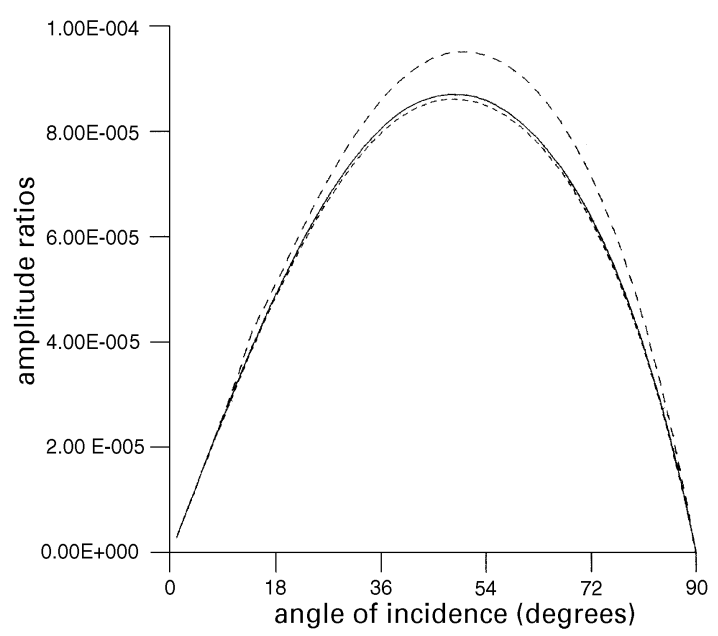

Figure 10. Variations of the modulus of amplitude ratios with the angle of incidence, for reflected modified CDII waves. 


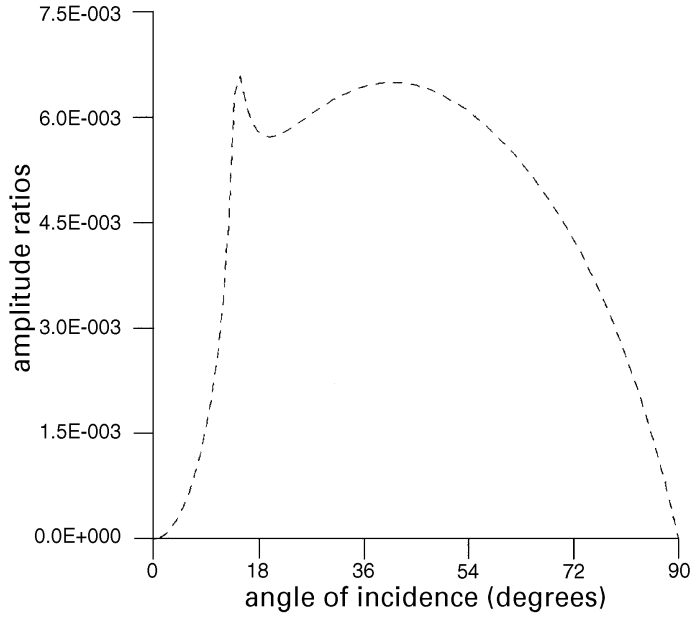

Figure 11. Variations of the modulus of amplitude ratios with the angle of incidence, for reflected LMS waves.

implies the importance of axial stretch phenomenon. Also, the micropolar elastic waves are modified due to viscous and stretch effects.

Numerical calculations in detail have been presented for the cases of longitudinal wave and modified longitudinal displacement wave incident at the interface of the model considered. From the numerical discussion, it may be concluded that stretch effect plays an important role in reflection and transmission phenomenon. Also, if we neglect the viscous effect, we find that the variations in reflected and refracted waves have also been affected but not as significantly as those due to the stretch effect. The model considered in the problem becomes more interesting due to inclusion of axial stretch and viscosity as additional parameters.

\section{References}

Eringen A C 1968 Theory of micropolar elasticity, fracture (New York: Academic Press) vol. 2

Eringen A C 1971 Micropolar elastic solids with stretch. Ari. Kitabevi Matabaasi 24: 1-9

Eringen A C, Suhubi E S 1964a Nonlinear theory of micro-elastic solids I. Int. J. Eng. Sci. 2: 189-203; Erigen A C, Suhubi E S 1964b Nonlinear theory of micro-elastic solids. Int. J. Eng. Sci. 2: 389-404

Eringen A C 1967 Linear theory of micropolar viscoelasticity. Int. J. Eng. Sci. 5: 191-204

Gauthier R D 1982 Experimental investigations on micropolar media. Mechanics of micropolar media (eds) O Brulin and R K T Hsieh (Singapore: World Scientific) p 395

Kumar R, Gogna M L, Debnath L 1990 On Lamb's problem in micropolar viscoelastic half-space with stretch. Int. J. Math. Sci. 13: 363-372

McCarthy M F, Eringen A C 1969 Micropolar viscoelastic waves. Int. J. Eng. Sci. 7: 447-458

Parfitt V R, Eringen A C 1969 Reflection of plane waves from the flat boundary of a micropolar elastic half-space. J. Acoust. Soc. Am. 45: 1258-1272

Tomar S K, Gogna M L 1992 Reflection and refraction of longitudinal microrotational wave at an interface between two different micropolar elastic solids in welded contact. Int. J. Eng. Sci. 30: $1637-1646$

Tomar S K, Gogna M L 1995 Reflection and refraction of coupled transverse and microrotational wave at an interface between two different micropolar elastic solids in welded contact. Int. J. Eng. Sci. 33: 485-492

Tomar S K, Kumar R 1995 Reflection and refraction of longitudinal displacement wave at a liquidmicropolar solid interface. Int. J. Eng. Sci. 33: 1507-1515 